\title{
¡†rECI\&NE
}

\section{Separador de fragmentos (F.R.S.) como herramienta para el análisis de reacciones nucleares a FAIR (Facility for Antiproton and Ion Research in Europe)}

\author{
Fragment separator (F.R.S.) as a tool for the analysis of nuclear reactions at FAlR \\ (Facillity for Antiproton and lon Research in Europe)
}

María Fernanda Robles-Cifuentes', Jossitt Williams Vargas-Cruz@2

'Servicio Geológico Colombiano, Bogotá, Colombia. mariferroci13@gmail.com;

2 Universidad Santo Tomás, Tunja, Colombia. jossittv@gmail.com

Recibido: 10 de febrero de 2020 Aceptado: 23 de junio de 2020

Resumen- Cada día la ciencia ha tenido que recurrir a la construcción de grandes instalaciones e instrumentos para realizar experimentos que permitan obtener información para que posteriormente sea analizada. En la física nuclear la creación de nuevas instalaciones ha venido creciendo a enormes pasos, ya que para estudiar esta área de la física ha sido necesario construir aceleradores, espectrómetros magnéticos y detectores con el objetivo de obtener información experimental de sucesos a escala microscópica. En el presente artículo se hace una revisión acerca del proceso del diseño y construcción del espectrómetro de alta resolución magnética SUPERFRS (Super-Fragment Separator) es resultado de una gran colaboración llamada FAIR en Darmstadt, Alemania, en las instalaciones del GSI, con el fin de conocer la motivación científica que llevó a construirlo, su diseño técnico y trabajos que se han de realizar en él.

Palabras clave- Espectrómetro, FRS, Super- FRS, física nuclear, GSI, FAIR.

Abstract- Science has had to resort to the construction of large facilities and instruments to carry out experiments to obtain information that can be analyzed later. In nuclear physics, the creation of new facilities has been growing enormously because, in order to study this area of physics, it has been necessary to build accelerators, magnetic spectrometers and detectors in order to obtain experimental information of events on a microscopic scale. This article reviews the design and construction process of the SUPER-FRS (Uper-Fragment Separator) magnetic high-resolution spectrometer resulting from a great collaboration called FAIR in Darmstadt (Germany) in the GSI facilities with a view to getting to know the scientific motivation that led to its construction, technical design and the work that has been done on it. FAIR.

Keywords- Spectrometer, FRS, Nuclear Physics, GSI,

\section{INTRODUCCIÓN}

En la física encontramos muchas áreas de investigación, como: física teórica, materia condensada, física atómica, física de altas energías, astrofísica, biofísica y física nuclear. En el contexto del presente artículo se enfocará especialmente a presentar algunos tópicos de física nuclear que es la rama de la física que se encarga del estudio del núcleo atómico y sus principales propiedades, así como estructura nuclear, modos de desintegración radioactiva, las interacciones entre constituyentes hadrónicos y reacciones nucleares [1-4].

Citar este artículo como: Robles-Cifuentes, M. F., \& Vargas-Cruz, J. W. Separador de fragmentos (F.R.S.) como herramienta para el análisis de reacciones nucleares a FAIR (Facility for Antiproton and Ion Research in Europe). ITECKNE, 17(2), 2020 pp. 83 - 90. doi: https://doi.org/10.15332/iteckne.v17i2.2467 
El entendimiento del núcleo atómico llevó a encontrar que en el sistema nuclear son relevantes tres de las cuatro fuerzas fundamentales de la naturaleza, a saber: la fuerza fuerte o nuclear, electromagnética y débil. En el siglo XX se logró un gran desarrollo de la física nuclear, pero aún no se ha logrado una teoría aceptable que describa las propiedades de los núcleos, a pesar de que la fuerza fuerte es un fenómeno ampliamente estudiado [5]. Sin embargo, se ha desarrollado una moderna teoría que explica la interacción fuerte llamada la Cromodinámica Cuántica (Quantum Chromodynamics Q.C.D.) [5-7].

Esta teoría ha sido verificada por experimentos que se realizaron en las instalaciones del CERN (Conseil Européen pour la Recherche Nucléaire) [8-9]. Debido a este hecho, el estudio de la física nuclear se realiza de manera iterativa, teniendo tres objetivos en su realización, como lo son: escrutar las partículas y sus interacciones dando campo a la física de partículas; clasificar e interpretar las propiedades de los núcleos y producir avances tecnológicos que beneficien a la sociedad [10-15]. Pero todo esto solo es posible con la colaboración de varias naciones, con el fin de grandes infraestructuras e instrumentos científicos para la realización de experimentos enfocados en indagar sobre la estructura básica de la materia.

En la actualidad, a nivel mundial, se están construyendo aceleradores distribuidos por todo el mundo. Sin embargo, el incipiente aumento de la complejidad, tanto de los experimentos como de los aceleradores, y por tanto de su costo económico, está dando lugar a un proceso de concentración en puntos muy localizados de la tierra, cuyo resultado es la creación de un reducido número de grandes centros de carácter internacional [16-17]. Un claro ejemplo lo constituye FAIR (Facility for Anti-proton and Ion Reseach) [18], cuyo fin es la investigación de la estructura atómica utilizando iones pesados a energías relativistas. También se construyen muchos aceleradores dedicados a proyectos aplicados, por citar dos de los casos más emblemáticos podemos mencionar el Tadem, ubicado en el laboratorio de los sótanos del Louvre, dedicado al análisis de obras de arte, o el Sincrotrón, recientemente instalado en el hospital de Heidelberg para terapia de tumores con iones pesados. En España hay dos aceleradores dedicados principalmente al estudio de materiales y al análisis arqueológico en el Centro Nacional de Aceleradores en Sevilla [19] y en la Universidad Autónoma de Madrid [20]; además, España cuenta con el acelerador de partículas más potente de Europa, el sincrotrón tiene por nombre ALBA y el cual es usado para la investigación de la física de la materia condensada [21].

En el presente artículo se describe de manera general el Fragment Separator (FRS) que es un espectrómetro magnético de alta resolución, ubicado en las instalaciones del GSI, Darmstad Alemania, y su entendimiento nos llevará a comprender la creación del Super-FRS el que sirve como plataforma y punto de referencia para la construcción de las instalaciones de FAIR.

\section{COMPAÑÍA PARA LA INVESTIGACIÓN DE IONES PESADOS (GSI)}

La Gesellshaft four Schwerionenforschung, en castellano "Compañía para la investigación de iones pesados", es una instalación científica radicada en Darmstadt (Alemania), que se dedica a la investigación y el desarrollo de tecnologías, principalmente en las áreas de la física, química y medicina [18].

Los resultados más conocidos de la investigación en el GSI son el descubrimiento de seis nuevos elementos químicos de la tabla periódica y el desarrollo de un novedoso tratamiento contra el cáncer con iones, entre estos esta: bohrium (1981) [22], meitnerium (1982) [23], hassium (1984) [24], darmstadtium (1994) [25], roentgenium (1994) [26], y copernicium (1996) [27]. Elementos cuya existencia está confirmada en GSI: Ununtrium (2012), Flerovium (2009), Ununpentium (2012), Livermorium (2010), Ununseptium (2012), y Ununoctium [28]. Los investigadores de todo el mundo utilizan las instalaciones del GSI para hacer experimentos con iones pesados, acelerados con el fin de indagar en aplicaciones en física de partículas, nuclear, física del plasma y los materiales de investigación para la biofísica y la terapia de tumores.

Como se puede observar en la Fig. 1 las instalaciones del antiguo GSI seis lugares que son indispensables para la ejecución y desarrollo de los experimentos con iones pesados en las instalaciones del GSI, estos lugares son: fuentes de iones, donde se generan las partículas antes de que se aceleren en UNILAC y SIS [29].

Fig. 1. VISTA ESQUEMÁTICA DE LA SALA EXPERIMENTAL GSI (DARMSTAD, ALEMANIA).

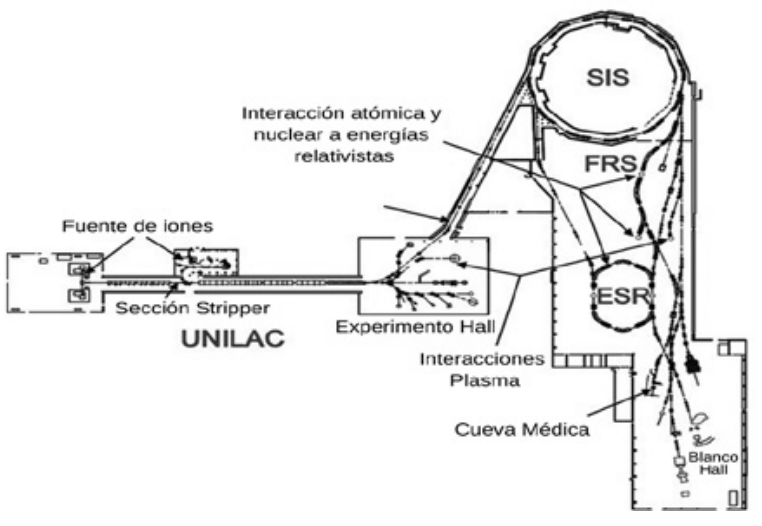

Fuente: Tomado de [35]

El acelerador lineal UNILAC (Universal Linear Accelerator) es el punto de partida de la aceleración de iones, tiene una longitud de 120 metros, además los iones de todo tipo pueden acelerarse hasta un 20 por ciento de la velocidad de la luz $(60.000 \mathrm{~km} / \mathrm{s})$ y estos iones se utilizan para crear nuevos elementos pesados, o se inyectan en el anillo acelerador SIS18 para una mayor aceleración [30], el anillo del 
acelerador SIS18, tiene como función proporcionar una mayor aceleración y después de que los iones han alcanzado la velocidad deseada se transfieren a los experimentos para golpear una muestra de material, o se dirigen al anillo de almacenamiento experimental ESR para su almacenamiento y análisis adicional [31], el anillo de almacenamiento ESR, en él permite que los iones puedan circular a altas velocidades, realizando varios millones de circulaciones por segundo, estos se almacenan y se utilizan posteriormente para experimentos [32], el Separador de Fragmentos FRS permite experimentar con nuevas partículas, especialmente con isótopos nuevos y extremadamente raros además de separar e identificar isótopos que se generan por una reacción [33] y Sala de Control Principal desde la cual controlan todos los 2500 componentes individuales controlables eléctricamente, como imanes, bombas de vacío e instrumentos de medición. Simplemente, los ingenieros de la instalación no podrían ajustar individualmente todos estos componentes a mano. Es por lo que las señales de todos los instrumentos se juntan en la sala de control principal [34]. La aceleración de los iones se produce con campos eléctricos elevados. A través de un campo magnético las partículas son preaceleradas y enfocadas. Tienen un alcance de velocidad máxima de alrededor del 90 por ciento de la velocidad de la luz.

\section{SEPARADOR DE FRAGMENTOS (FRS)}

El separador de fragmentos (Fragment eparator FRS) [36-37] es un espectrómetro magnético de alta resolución, capaz de separar e identificar núcleos a energías relativistas producidos en una reacción nuclear.

La motivación científica para la construcción del FRS fue la planeación de experimentos con especial énfasis en la producción de especies de núcleos por el método de separación electromagnética. Inicialmente se realizaron experimentos en áreas como la física de partículas, física nuclear, en la aplicación de rayos de radiación relativistas y otros campos como la biofísica y la física médica. En la actualidad los experimentos que se realizan en el FRS con nuevas partículas, especialmente con isótopos nuevos o extremadamente raros, permiten crear nuevas partículas (o isótopos) por reacciones especiales de iones previamente acelerados en el SIS18, que es un acelerador circular tipo Álvarez [38]. Después de la etapa de preaceleración los isótopos interactúan con un blanco de reacción y luego los productos de la reacción se direccionan al FRS donde se pueden separar de acuerdo con su relación carga-masa. Para algunas aplicaciones, los productos de reacción se pasan entonces a la ESR anillo de almacenamiento o se utilizan para experimentos adicionales [39-41].

En la Fig. 2 se muestra un esquema de todos los planos focales del FRS. El separador de fragmentos tiene cuatro sistemas independientes denominados planos focales. Cada plano focal está constituido por un dipolo magnético a 30 grados y un conjunto de cuatro dipolos y de sextupolos que corrigen efectos de aberración de óptica magnética.

\section{Fig. 2. ESQUEMA DE LOS PLANOS FOCALES DEL FRS}

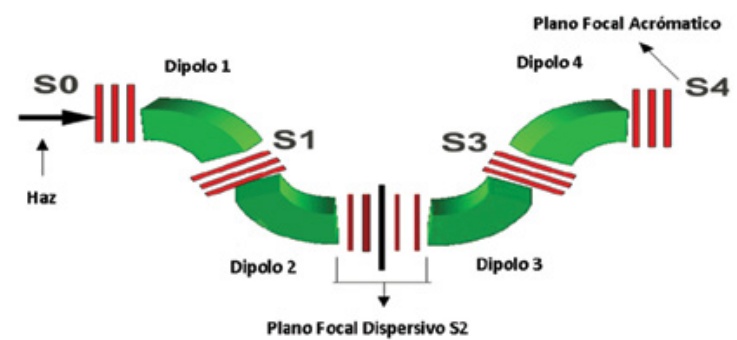

Fuente: Tomado de [42]

El FRS tiene dos modos de operación, se puede en modo de pérdida de energía y en acromático. Cuando se usa el espectrómetro en modo de pérdida de energía, el haz precisa medidas de transferencia de energía en el núcleo, en este modo de operación el blanco se coloca en el centro del plano focal.

En modo acromático el FRS se ajusta de tal manera que el momentum sea independiente del ángulo, mejorando la precisión en el espacio de momentos. Los dos primeros planos focales del FRS están ubicados a diferentes distancias para lograr una resolución máxima de momentum en los planos focales dispersivos. La distancia correspondiente al primer plano es de $2.20 \mathrm{~m}$. El segundo plano focal está situado a $0.90 \mathrm{~m}$ y permite una mayor aceptancia, que es la capacidad que tiene el espectrómetro de ampliar el rango de análisis isotópico, a expensas de la resolución de momento [36]. Las ramas de investigación del FRS están dedicadas a diferentes experimentos, los cuales son:

Una rama baja de energía para los estudios de desintegración y captura de iones. Y una rama en un sistema de dos anillos para los experimentos de precisión con almacén y termalización exótica de núcleos [41].

\section{Instalación para la investigación de antiprotones e iones en Europa (FAIR)}

La Facility For Antiprotón And Ion Research in Europe, en castellano "Instalaciones para la investigación de antiprotones e iones en Europa", es uno de los proyectos de investigación más grandes del mundo, y en la actualidad se está construyendo en el campus del GSI en la ciudad de Darmstadt, Alemania [43], y a su vez utilizará algunos de los componentes del acelerador de GSI como sistemas de inyección. Rayos de iones y antiprotones de alta intensidad serán utilizados por diferentes grupos internacionales de investigación para realizar experimentos, desde sondeo de quarks hasta átomos. El nuevo acelerador entregará haces de iones y antiprotones de alta intensidad para experimentos en los campos de física atómica, física del plasma, física nuclear, física de hadrones, física de materiales y biofísica con aplicaciones hacia nuevos tratamientos médicos y ciencia espacial [45]. En estos experimentos participarán más de 2500 científicos e ingenieros de más de 50 países 
de los 5 continentes Las instalaciones de FAIR se espera que comience a funcionar a partir del año 2025 [43-46].

El corazón de FAIR es el sincrotrón de iones pesados de aceleración rápida SIS100. Esta máquina de alta intensidad se complementa con un acelerador lineal de protones utilizados para la producción de antiprotones El acelerador de FAIR es único y complejo al ofrecer haces de todas las especies de iones y antiprotones a altas energías con altas intensidades y calidad sin precedentes, en otras palabras ofrece haces con energía muy precisa y perfiles de haces colimados [45-48].

Una de las más grandes y completas disposiciones experimentales en FAIR es el super separador de fragmentos superconductores (SFRS) para la generación e identificación limpia de haces secundarios de iones de corta duración.

Como se puede observar en la figura 3, la nueva instalación y el complejo GSI existente se muestran en rojo y gris, respectivamente, junto a las nuevas construcciones de FAIR. En el corazón de FAIR hay un túnel que alberga dos anillos aceleradores con un poder de flexión de $100 \mathrm{Tm}$ y 300 Tm, llamados SIS-100 y SIS-300. EI MSV de FAIR incluye el anillo SIS-100, objetivos de producción para haces de antiprotones y haces secundarios (estable y de corta duración), el anillo de almacenamiento de alta energía (HESR) para almacenar los haces antiprotones y el super separador de fragmentos superconductores (SFRS) para haces de iones secundarios. La versión completa de la instalación incluirá el anillo SIS-300 que proporcionará operación paralela de experimentos junto con una mayor energía del haz.

Fig. 3: Diseño general de las instalaciones de la FAIR

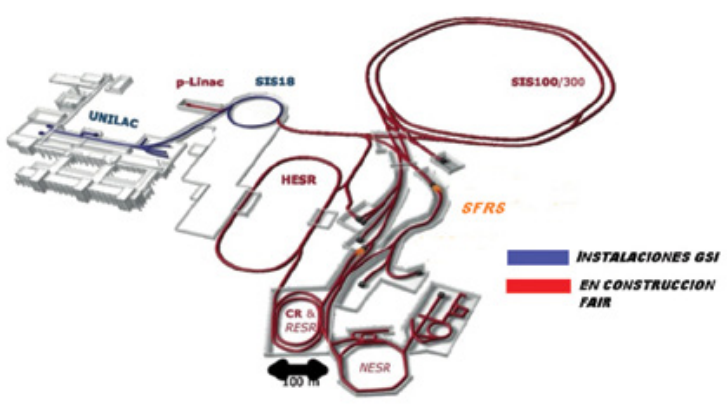

Fuente: tomado de [43-47].

En términos generales, las comunidades de investigación en FAIR se han dividido en cuatro categorías diferentes; es decir, (a) aplicaciones y física atómica y de plasma (APPA), (b) estructura nuclear, astrofísica y reacciones (NUSTAR) [49], el cual con experimentos con núcleos atómicos son la clave para comprender las estrellas, (c) física de hadrones utilizando haces anti-protónicos (PANDA) [50] y (d) el comprimido experimento de materia bariónica (CBM) [51-56] para crear y estudiar la materia que interactúa fuertemente bajo muy alta densidad neta de bariones. La colisión de núcleos atómicos a altas velocidades puede simular las condiciones dentro de objetos supermasivos durante una fracción de segundo. La fase de inicio conocida como la versión de inicio modular (MSV) está planificada de tal manera que las cuatro comunidades pueden comenzar sus experimentos en las respectivas fases de inicio desde el comienzo de la operación de FAIR [46].

\section{SUPER SEPARADOR DE FRAGMENTOS SUPERCONDUCTORES (SFRS)}

El SFRS (Super Fragment Separator Superconductor SFRS) es uno de los principales instrumentos científicos de las futuras instalaciones de FAIR en Darmstadt, Alemania. Será un espectrómetro magnético de alta resolución, acoplado al sincrotrón de iones pesados (SIS-18 / SIS-100), y sin duda es el protagonista de una de las más importantes colaboraciones que se llevarán a cabo en FAIR, la colaboración para la investigación con núcleos exóticos NUSTAR [57-59].

El Super-FRS se describe como una instalación única en el mundo en comparación con otros espectrómetros que existen o se estén planificando, es debido a sus propiedades únicas, las cuales se pueden destacar:

1. En el Super-FRS, se pueden usar haces nucleares primarios y secundarios de alta energía y alta intensidad con energías de hasta 1.500 A MeV.

2. Es un espectrómetro único de alta energía y alta resolución con una gran aceptancia y flexibilidad en los ajustes en la óptica iónica, en particular con capacidades de coincidencia de dispersión.

3. Proporciona un alto poder de supresión del haz primario y un alto poder de separación de núcleos de hasta $Z=92$, y también proporciona iones completamente despojados de todos los elementos.

4. Proporciona modos de espectrómetro versátiles mediante diferentes combinaciones de secciones de separación [60].

El Super-FRS proporcionará haces de núcleos exóticos que van desde hidrógeno hasta uranio en un amplio rango de energía, equivalente a un máximo de rigidez magnética de hasta $20 \mathrm{Tm}$. Los núcleos exóticos se producen a través de fragmentación de proyectiles y reacciones de fisión. Los núcleos de interés serán separados por la técnica de separación de vuelo y entregados en varios cientos de nanosegundos y entregados a los sistemas de detectores a gran escala: planeados dentro de la versión de inicio modular de FAIR, que se colocarán en las tres salidas separadoras: HISPEC/DESPEC and MATS/LaSPEC fase de baja energía (LEB), R3B fase de alta energía Branch (HEB) y ILIMA fase de anillo de almacenamiento (RB). El alto poder de separación se logra bajo la condición de espacio de fase $40 \mathrm{~mm}$ mrad y una aceptancia de momento longitudinal de $\Delta p / p= \pm 2.5 \%$. $P[60]$. 
La Fig. 4 muestra el diseño del SFRS, el cual posee dos etapas de separación: el pre-separador y el separador principal. Ambas etapas del separador usan el Bq, método [3], donde se realiza un doble análisis de rigidez magnética aplicado delante y detrás de una energía especialmente inyectada. El separador principal tiene tres ramas terminadas por áreas experimentales dedicadas: la alta energía Rama (HEB) para estudios de reacción a energías relativistas, Ring-Branch (RB) para experimentos de precisión con núcleos exóticos almacenados y enfriados y de baja energía Branch (LEB) para espectroscopía y estudios de descomposición y también atrapamiento de iones. La salida del LEB está acoplada a una combinación de espectrómetro magnético y acumulador de energía, que es esencial para realizar experimentos de manera eficiente con ralentizado o incluso detenido iones [61].

Fig. 4. ESQUEMA GENERAL DEL SUPER-SEPARADOR DE FRAGMENTOS (SFRS)

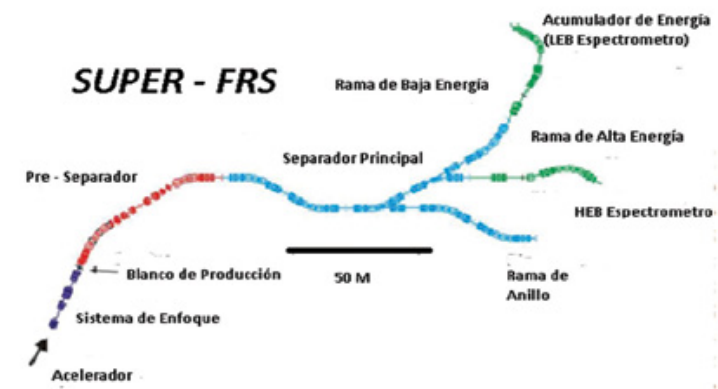

Fuente: Tomado de [60]

Las intensidades del haz primario más altas requieren una mayor pureza como resultado de la combinación de dos etapas de separación, donde cada etapa aplica el método rigidez magnética. En consecuencia, el preseparador y el separador principal están equipados con un sistema de degradación de energía. Las ventajas del sistema de dos degradadores pueden resumirse como: reducción de contaminantes de fragmentos, introducción de otro corte de separación en la $A-Z$, posible uso de pre y separador principal para estudios de reacción secundaria, optimización de la tasa de fragmentos en detectores en el separador principal [61].

\section{RAMAS DE INVESTIGACIÓN PARA LAS QUE ESTÁ PROYECTADO UTILIZAR EL SFRS}

Las ramas de investigación para las que está proyectado el SUPER-FRS están dedicadas a diferentes experimentos, los cuales se nombran a continuación:

5.1. Estudios de colisión atómica: El conocimiento preciso de la interacción atómica de iones pesados para penetrar a través de la materia es esencial, por ello es importante contar con un espectrómetro de masas de tiempo de vuelo de reflexión múltiple para la realización de mediciones de masa directa, para ello el espectrómetro FRS y proporcionar un haz isobáricamente limpio para experimentos pos- teriores, como la espectroscopia de desintegración seleccionada en masa, los cuales sirven como base para la operación exitosa de la Super-FRS $[18,19]$. En el dominio de la energía en el SFRS, para almacenar datos de potencias, energía y distribuciones en estado de carga de iones pesados son escasos. Por lo tanto, los procesos básicos de colisión atómica tienen que ser medidos en una etapa temprana de la operación Super-FRS. La altura, capacidades de resolución de momento y separación de estado de carga del Super-FRS sugiere la base sólida para tales estudios [62-64]

5.2. Las fuerzas tensoras en los núcleos: Cálculos recientes de núcleos de luz demuestran que aproximadamente el $80 \%$ de la unión nuclear se debe a los piones y la fuerza tensora es tan importante como las fuerzas centrales en la interacción de intercambio de piones, para este estudio se necesita experimentos de haces incidentes de alta energía superiores a 400 AMeV con un modo de espectrómetro de cero grados de alta resolución, siendo el SFRS la única instalación en el mundo que proporciona haces incidentes de estas energías [65-66].

5.3. Átomos piónicos: El descubrimiento de estados piónicos profundamente unidos en átomos pesados en FRS ha abierto un nuevo campo de estudios fundamentales de las interacciones mesón-núcleo, que contribuyen a la comprensión de la estructura no trivial del vacío del cromo cuántico dinámica (QCD) [67-68].

5.4. Radios nucleares y distribuciones de momento: Los experimentos propuestos combinan mediciones de secciones transversales de interacción y cambio de carga con nucleón eliminación y medida de distribución de momento de alta resolución ments. Dichos estudios están dirigidos a nuevas estructuras en núcleos, en partículas en regiones de masa más pesada $(A>50)$ [69].

\section{CONCLUSIONES}

Las instalaciones de investigación a nivel mundial enfocadas a estudiar fenómenos de la física nuclear, atómica, de plasma, etc., están en un continuo cambio, ya que al ir indagando más profundo en la estructura interna del núcleo más retos experimentales salen a la vista.

El primer paso en las investigaciones a nivel nuclear y de partículas, es invertir en experimentos enfocados a solucionar problemas planteados en las ciencias básicas que en un futuro se propenderá a la creación de la tecnología del mañana.

Es por lo que investigaciones que al comienzo parecían ser descabelladas, ahora son la tecnología actual y de punta; es decir, la investigación fundamental de hoy será la tecnología del mañana. 
Dentro de los productos de las diferentes investigaciones que ha dado a conocer el GSI al mundo, se encuentran por ejemplo el descubrimiento de seis nuevos elementos químicos y un nuevo tipo de terapia tumoral basada en la irradiación con iones.

El GSI, no solo ha generado nuevos conocimientos y tecnología, sino que también ha contribuido en la formación científica de varios profesionales de distintas universidades e instituciones de todo el mundo, ya que en sus laboratorios existe la posibilidad de ser científico visitante y contribuir en las investigaciones que adelantan, y de esta manera convirtiéndose en un centro de formación de las nuevas generaciones de físicos nucleares.

El SFRS es una herramienta tremendamente potente para estudiar reacciones nucleares a alta y bajas energías, ya que el amplio espectro de rigideces magnéticas obtenido en SFRS combinado con una muy precisa eliminación de contaminantes de reacciones nucleares, permitirá estudiar secciones eficaces y variables dinámicas, como las distribuciones de cuadrimomento con mayor precisión. Este tipo de investigaciones ha impulsado avances en astrofísica, física médica, biología, ciencia de aceleradores, entre otras directas aplicaciones.

Los investigadores en Latinoamérica pueden participar de manera indirecta, contactando grupos que trabajen directamente en GSI con el objetivo de proponer, simular y a futuro analizar nuevos proyectos enmarcados en el plan de trabajo de PANDA, NUSTAR, APPA, CBM y demás colaboraciones con injerencia en FAIR, ya que existen muchísimos problemas, ya sea a nivel teórico y experimental que merecen de nuestra comprensión y que la comunidad científica mundial estará presta a estudiar.

\section{REFERENCIAS}

[1] E. Epelbaum, H.W. Hammer, \& U.G. Meißner, Modern theory of nuclear forces. Reviews of Modern Physics, 81 (4), 2009 pp. 1773.

[2] J.M. Arias, M. Lozano, "Advanced course in modern nuclear physics," Lecture Notes in Physics, 1st Edition, Ed Springer, 2010.

[3] Vena Carter, Advanced Nuclear Physics, Ed. Global Media, City, 2009, pp. 104.

[4] J.W. Negele, Erich W. Vogt. Advances in Nuclear Physics, Advances in the Physics of Particles and Nuclei, 23 ed. Vol, ed Springer, 1996 pp. 315.

[5] P. Sokolsky, P. Sommers, \& B.R. Dawson, Extremely high energy cosmic rays. Physics reports, 217 (5), 1992 pp. 225-277.

[6] S. Dürr, Z. Fodor, J. Frison, C. Hoelbling, R. Hoffmann, S.D. Katz, \& K.K. Szabo, Ab initio determination of light hadron masses. Science, 322 (5905), 2008 pp. 1224-1227.

[7] A. S. Kronfeld, Journal of Physics: Conference Series 125, 2008,01206
[8] J. Casaus, J. Rodríguez y E. Sánchez, Cromodinámica Cuántica: el color de los quarks, Departamento de Fusión y Física de Partículas Elementales. CIEMAT, 2005.

[9] K. A. Olive, "Review of particle physics," Chinese physics C, 38(9), 2014, 090001-090001.

[10] A. F. Soria, Física nuclear y de partículas, Vol. 62, 2015, Universitat de València.

[11] R. Stock, Encyclopedia of nuclear physics and its applications, 2013, John Wiley \& Sons.

[12] R. J. Blin-Stoyle FRS, Physics and Its Applications, ed. Springer Netherland, ed 1, 1991 pp. 224.

[13] K. Bethge, G. Kraft, P. Kreisler, G. Walter, Medical Applications of Nuclear Physics, Biological and Medical Physics, Biomedical Engineering, Springer-Verlag, ed 1, 2004 pp. 209.

[14] J. Lilley, Nuclear physics: principles and applications, 2013, John Wiley \& Sons.

[15] J. Thomas, Material characterization using ion beams, Vol. 28, 2012, Springer Science \& Business Media.

[16] F. Lambert, Y.Volkov, “NATO Science for Peace and Security Series B: Physics and Biophysics", ed 1, 2006 pp. 217.

[17] J. Benlliure y B. Rubio, "Grandes instalaciones científicas en física nuclear: pasado, presente y futuro," [Online] REF Enero-marzo 2008, Temas de Física Available: http:// www.rsef.org,

[18] GSI, GSI, 2020, www.gsi.de/fair

[19] B. M. Nyako, et al. Phys. Rev. Lett, 52 (1984) 507 y P.J. Twin, et al. Phys. Rev. Lett, 54, 1986 pp. 811.

[20] Laboratori Nazionali di Legnaro, Institu Nacionale di Fisica Nucleare, 2018, http://www.Inl.infn.it/index.php/en/

[21] P. Bolufer, AlbA. In Anales de mecánica y electricidad, 2010.

[22] R. Malmbeck, G. Skarnemark, J. Alstad, K. Fure, M. Johansson, \& J. Omtvedt, "Chemical separation procedure proposed for studies of bohrium," Journal of Radioanalytical and Nuclear Chemistry, 246 (2), 2000, pp. 349-353.

[23] Journal of Radioanalytical and Nuclear Chemistry, Vol. 246, Issue 2, 2000, pp. 349-353.

[24] C. E. Düllmann. Superheavy elements at GSI: a broad research program with element 114 in the focus of physics and chemistry. Radiochimica Acta, 100 (2), 2012 pp. 67-74.

[25] J. Dvorak, W. Bruchle, M. Chelnokov, C.E. Dullmann, Z. Dvorakova, K. Eberhardt, \& Z. Qin, Doubly magic nucleus 270Hs, 2008.

[26] C. E. Düllmann, "Superheavy elements at GSI: a broad research program with element 114 in the focus of physics and chemistry". Radiochimica, Acta 100 (2), 2012 pp. 67-74.

[27] K. Morita, "Decay of an Isotope 277112 produced by 208Pb+ 70Zn reaction," In Exotic Nuclei Exon2004, 2005 pp. 188-191.

[28] H. Geissel, P. Armbruster, K.H. Behr, Nuclear Instruments and Methods in Physics Research Section B: Beam Interactions with Materials and Atoms, Vol. 70, Issues 1-4, 1 August, 1992, pp. 286-297.

[29] H. Geissel, P. Ambruster et al., A proposal for the SIS-ESR, experimental Program, 1987. 
[30] K. Blasche, SIS Beam Development 1981-1991, GSIReport GSI-SIS-INT/86-2.

[31] GSI, GSI ACELERATOR, 2020, https://www.gsi.de/en/ researchaccelerators/accelerator_facility/ion_sources starting_point_of_the_gsi_accelerator_facility_ion_ sources.htm]

[32] GSI. GSI ACELERATOR, 2020, https://www.gsi.de/ en/researchaccelerators/accelerator_facility/linear_ accelerator.htm

[33] GSI. GSI ACELERATOR, 2020, https://www.gsi.de/ en/researchaccelerators/accelerator_facility/ring_ accelerator.htm.

[34] GSI. GSI ACELERATOR, 2020, https://www.gsi.de/en/ researchaccelerators/accelerator_facility/storage_ring. htm

[35] A. Fettouhi,"Investigation of Slowing Down and ChargeExchange of Nickel and Uranium Ions in Gases and Solids in the Energy Range (60-200) MeV/u", (Doctoral dissertation), 2006.

[36] GSI. GSI ACELERATOR, 2020, https://www.gsi.de/en/ researchaccelerators/accelerator facility/main control room.htm.

[37] GSI. GSI ACELERATOR, 2020, https://www.gsi.de/en/ start/news.htm

[38] H. Geissel, P. Armbruster, K.H. Behr, A. Brünle, K. Burkard, M. Chen, ... \& B. Langenbeck, "The GSI projectile fragment separator (FRS): a versatile magnetic system for relativistic heavy ions," Nuclear Instruments and Methods in Physics Research Section B: Beam Interactions with Materials and Atoms, 70 (1-4), 1992 pp. 286-297.

[39] F. Bosch, First experiments at the Darmstadt storagecooler ring ESR. Nuclear Instruments and Methods in Physics Research Section A: Accelerators, Spectrometers, Detectors and Associated Equipment, 314 (2), 1992 pp. 269-276.

[40] H. Geissel, P. Armbruster, K. H. Behr, A. Brünle, K. Burkard, M. Chen, ... \& B. Langenbeck, The GSI projectile fragment separator (FRS): a versatile magnetic system for relativistic heavy ions. Nuclear Instruments and Methods in Physics Research Section B: Beam Interactions with Materials and Atoms, 70 (1-4), 1992 pp. 286-297.

[41] FAIR. FAIR, 2020, https://fair-center.eu/index.php?id=1

[42] V. Cruz, \& J. Williams, Charge exchange and knockout reactions induced by $\mathrm{Sn}$ isotopes at relativistic energies, 2014.

[43] GSI. GSI, 2020, https://www.gsi.de/en/ researchaccelerators/fair/fair

[44] M. Durante, P. Indelicato, B. Jonson, V. Koch, K. Langanke, U.G. Meißner, ... \& M. Wiescher, "All the fun of the FAIR: fundamental physics at the facility for antiproton and ion research," Physica Scripta, 94 (3), 2019, 033001.

[45] FAIR. Physics at FAIR, 2020, https://www.gsi.de/en/ researchaccelerators/fair/research.htm

[46] J. Steinheimer, I. Augustin, A. Andronic, T. Saito, P. Senger, \& $\mathrm{H}$. Stoecker, Strangeness at the international Facility for Antiproton and lon Research. Journal of Physics G: Nuclear and Particle Physics, 36 (6), 2009, 064036.
[47] A. Bracco, "The NuPECC long range plan 2017: perspectives in nuclear physics," Europhysics News, 48 (4), 2017 pp. 21-24.

[48] S. Chattopadhyay, "Physics at FAIR," Nuclear Physics A, 931, 2014 pp. 267-276.

[49] W. Erni, I. Keshelashvili, B. Krusche, M. Steinacher, Y. Heng, Z. Liu, ... \& J. Becker, "Physics performance report for PANDA: Strong interaction studies with antiprotons," arXiv preprint arXiv:0903.3905, 2009.

[50] W. Ehehalt, \&W. Cassing, "Relativistic transport approach for nucleus-nucleus collisions from SIS to SPS energies," Nuclear Physics A, 602 (3-4), 1996 pp. 449-486.

[51] M. Orsaria, H. Rodrigues, F. Weber, \& G. A. Contrera, "Quark deconfinement in high-mass neutron stars. Physical Review C, 89 (1), 2014 pp. 15806-15817.

[52] http://www2.yukawa.kyotou.ac.jp/ kenji.fukushima/ denseQCD/talks/Fukushima.pdf, arXiv:1301.6377.

[53] L. McLerran, "Quarkyonic matter and the phase diagram of QCD," Continuous Advances In QCD, 2008 pp. 125-134.

[54] FAIR. FAIR EXPERIMENTS, 2020, http://www.fair-center. eu/for-users/experiments/cbm.html.

[55] B. Friman, C. Höhne, J. Knoll, S. Leupold, J. Randrup, R. Rapp, \& P. Senger, The CBM physics book: Compressed baryonic matter in laboratory experiments, Vol. 814, 2011, Springer.

[56] J. Äystö, K. H. Behr, J. Benlliure, A. Bracco, P. Egelhof, A. Fomichev, ... \& M. N. Harakeh, Experimental program of the Super-FRS Collaboration at FAIR and developments of related instrumentation. Nuclear Instruments and Methods in Physics Research Section B: Beam Interactions with Materials and Atoms, 376, 2016 pp. 111-115.

[57] H. Geissel, L. Chen, T. Dickel, F. Farinon, I. Dillmann, R. Knöbel, ... \& Z. Patyk, "Recent results from FRS experiments with exotic nuclei produced with uranium projectiles and perspectives with the Super-FRS," Exotic Nuclei: EXON-2012 pp. 263-272.

[58] M. Winkler, H. Geissel, H. Weick, B. Achenbach, K. H. Behr, D. Boutin, ... \& A. Kelic, "The status of the Super-FRS inflight facility at FAIR," Nuclear Instruments and Methods in Physics Research Section B: Beam Interactions with Materials and Atoms, 266 (19-20), 2008 pp. 4183-4187.

[59] Scientific program of the Super-FRS Collaboration: Report of the collaboration to the FAIR management. Super-FRS Collaboration, 2016.

[60] M. Winkler, H. Geissel, H. Weick, B. Achenbach, K. H. Behr, D. Boutin, ... \& A. Kelic, The status of the Super-FRS inflight facility at FAIR. Nuclear Instruments and Methods in Physics Research Section B: Beam Interactions with Materials and Atoms, 266 (19-20), 2008 pp. 4183-4187.

[61] [61] C. A. Douma, J. Gellanki, M. A. Najafi, H. Moeini, N. Kalantar-Nayestanaki, C. Rigollet, ... \& H. J. Timersma, "Verification of passive cooling techniques in the SuperFRS beam collimators," Journal of Physics: Conference Series, Vol. 742, No. 1, 2016012031.

[62] W. R. Plaß, T. Dickel, S. Purushothaman, P. Dendooven, H. Geissel, J. Ebert, ... \& H. Weick, The FRS Ion Catcher-a facility for high-precision experiments with stopped projectile and fission fragments. Nuclear Instruments and Methods in Physics Research Section B: Beam 
Interactions with Materials and Atoms, 317, $2013 \mathrm{pp}$. 457-462.

[63] I. Mardor, O. Aviv, M. Avrigeanu, D. Berkovits, A. Dahan, T. Dickel, ... \& M. Hass, "The soreq applied research accelerator facility (saraf): overview, research programs and future plans,"The European Physical Journal A, 54(5), 2018 pp. 91.

[64] I. E. Tamm, \& I. M. Frank, "Coherent radiation of fast electrons in a medium," Dokl. Akad. Nauk SSSR, Vol. 14, No. 3, 1937 pp. 107-112.

[65] R. Tamagaki, “Potential models of nuclear forces at small distances," Progress of Theoretical Physics, 39(1) 1968 pp. 91-107.
[66] H. J. Ong, I. Tanihata, A. Tamii, T. Myo, K. Ogata, M. Fukuda, ... \& H. Matsubara, "Probing effect of tensor interactions in 160 via $(p, d)$ reaction," Physics Letters B, 725 (4-5), 2013 pp. 277-281.

[67] T.Yamazaki, R. S. Hayano, K. Itahashi, K. Oyama, A. Gillitzer, H. Gilg, ... \& H. Geissel, "Discovery of deeply bound $\pi-$ states in the $208 \mathrm{~Pb}(\mathrm{~d}, 3 \mathrm{He})$ reaction," Zeitschrift für Physik A Hadrons and Nuclei, 355(3), 1996 pp. 219-221.

[68] K. Itahashi, “Letter of Intent for GSI-SIS”, 2011; K. Itahashi et al. Prog. Theor. Phys, 128, 2012 pp. 601.

[69] R. Kanungo, "A new view of nuclear shells," Physica Scripta, T152, 2013, 014002. 